\title{
KHK-Prävention: Sprints genauso wirksam wie Joggen
}

\section{Sport schützt vor Herz-Kreislauf- Krankheiten. Forscher der University of The West of Scotland in Hamilton (UK) wollten wissen, ob kurze Belas- tungen, etwa Sprints, genauso ef- fektiv sind wie Jogging über längere Zeit.}

- Sie untersuchten Freiwillige im Schulkindalter $(16,4+0,7$ Jahre), insgesamt 47 Knaben und zehn Mädchen. Die Kinder wurden nach dem Zufallsprinzip in drei Gruppen aufgeteilt: Gruppe 1 mit mäßigem Ausdauertraining (12 Knaben, 4 Mädchen) - Joggen in lockerem, gleichmäßigem Tempo mit $70 \%$ der maximalen $\mathrm{O}_{2}$-Aufnahme). Gruppe 2 trainierte im hochintensivem Bereich (15 Knaben, 2 Mädchen), 20-Meter Sprints. Die Sportstunden fan-

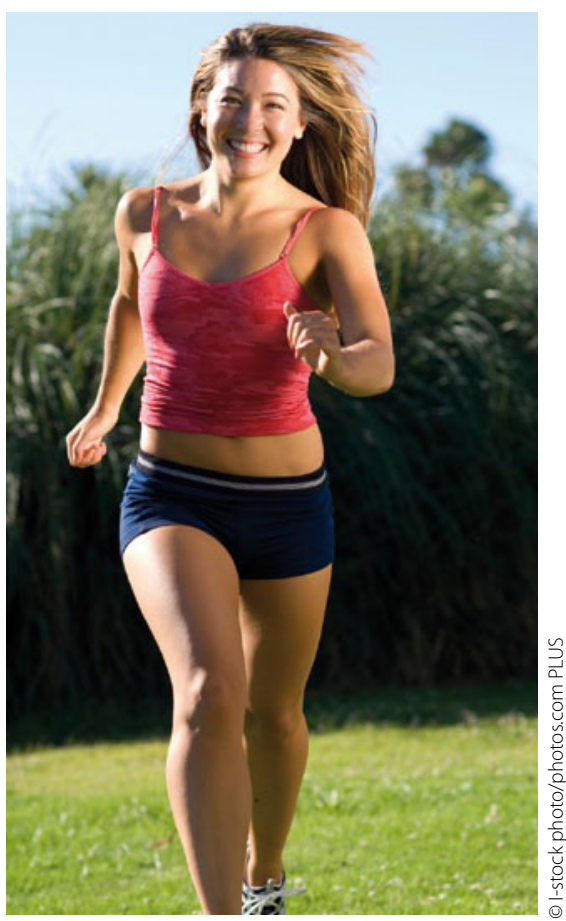

Wer schneller läuft, muss weniger Zeit für das Kreislauf-Training investieren. den dreimal pro Woche über sieben Wochen statt. Der Rest der Kinder bildete die Kontrollgruppe (Gruppe3).

Durch den Sport sank für beide Gruppen das Herzrisiko. Unabhängig von den Trainingsintensitäten wurden die Werte für Herz und Lunge, Blutdruck und Insulinresistenz besser ( $\mathrm{p} \leq$ $0,05)$. Aber der Zeitbedarf war geringer. Während die Jogginggruppe 420 Minuten trainierte, benötigte die Sprintgruppe nur 63 Minuten.

\section{Kommentar}

Zwar waren die Untersuchungsgruppen klein, die Ergebnisse waren aber einheitlich. Gleichgütig mit welcher Trainingsintensität gearbeitet wurde, die kreislaufreleventan Parameter der Teenager besserten sich. Damit sind kurze intensive Trainingseinheiten für Kinder und Jugendliche (und wohl nicht nur für die) eine gute Wahl, zeiteffektiv zu trainieren. Die Risikofaktoren für Herzkreislauferkrankungen, die ihren Ursprung bereits in der Kindheit haben, können effektiver bekämpft werden.

K. MALBERG

- D. S. Buchan et al.

(Health and Exercise Sciences, School of Science, University of the West of Scotland, Hamilton, ML3 0JB, Scotland, UK; E-mail:duncan. buchan@uws.ac.uk) The effects of time and intensity of exercise on novel and established markers of CVD in adolescent youth. First published online: 4 APR 2011; Amer. J. Human. Biol. 23: doi: 10.1002/ajhb.21166 